\title{
MAXIMAL SOLUTIONS TO FRACTIONAL DIFFERENTIAL EQUATIONS
}

\author{
Christopher C. Tisdell
}

\begin{abstract}
When do fractional differential equations have maximal solutions? This note discusses this question in the following way. Firstly, a comparison theorem is formulated that involves fractional differential inequalities. Secondly, a sequence of approximative problems involving polynomials is analyzed and it is shown that there is a subsequence of solutions whose limit is the maximal solution to the original problem of interest. In particular, the interval of existence for the maximal solution is the optimal length, aligning with best practice in the local theory of existence of solutions. We achieve this through an application of the Arzela-Ascoli Theorem and our aforementioned comparison result. A YouTube video by the author designed to complement this work is available at http://tinyurl.com/MaxFracDE.
\end{abstract}

Mathematics subject classification (2010): 34A08, 34A12.

Keywords and phrases: Maximal solution, comparison theorem, existence of solutions, fractional differential equations, initial value problem.

\section{REFERENCES}

[1] Michele CAPUto, Linear models of dissipation whose $Q$ is almost frequency independent, II, (English summary) Reprinted from Geophys. J. R. Astr. Soc. 13 (1967), no. 5, 529-539. Fract. Calc. Appl. Anal. 11, 1 (2008), 4-14.

[2] Kai Diethelm, Neville J. Ford, Analysis of fractional differential equations, J. Math. Anal. Appl. 265, 2 (2002), 229-248.

[3] Kai Diethem, The analysis of fractional differential equations, Springer, Heidelberg, 2010.

[4] Anatoly A. Kilbas, Hari M. SRivastava, Juan J. Trujillo, Theory and applications of fractional differential equations, North-Holland Mathematics Studies, 204. Elsevier Science B. V., Amsterdam, 2006.

[5] V. Lakshmikantham, A. S. Vatsala, Theory of fractional differential inequalities and applications, Commun. Appl. Anal. 11, 3-4 (2007), 395-402.

[6] V. Lakshmikantham, A. S. Vatsala, General uniqueness and monotone iterative technique for fractional differential equations, Appl. Math. Lett. 21, 8 (2008), 828-834.

[7] V. Lakshmikantham, A. S. Vatsala, Basic theory of fractional differential equations, Nonlinear Anal. 69, 8 (2008), 2677-2682.

[8] W. Rudin, Principles of mathematical analysis, McGraw-Hill, New York, 1964.

[9] C. C. TISDELL, On the application of sequential and fixed-point methods to fractional differential equations of arbitrary order, J. Integral Equ. Appl. 24, 2 (2012), 283-319.

[10] C. C. TISDELL, Solutions to fractional differential equations that extend, J. Classical Anal. 5, 2 (2014), $129-136$.

[11] C. C. TISDELL, When do fractional differential equations have solutions that are bounded by the Mittag-Leffler function?, Fract. Calc. Appl. Anal. 18, 3 (2015), 642-650.

[12] Chris Tisdell, Gronwall's inequality and fractional differential equations, Dr Chris Tisdell's YouTube Channel, 06/09/2011, accessed 25/06/2014, www . youtube . com/watch?v=Ixags $16 \mathrm{MWxI}$.

[13] Chris Tisdell, Approximate solutions to fractional differential equations, Dr Chris Tisdell's YouTube Channel, 08/09/2011, accessed 25/06/2014, www . youtube . com/watch?v=d5tZ7M7c_Y4.

[14] Chris Tisdell, Banach fixed point thoerem and differential equations, Dr Chris Tisdell's YouTube Channel, 08/09/2011, accessed 25/06/2014, www . youtube. com/watch?v=RhAjh8QpkDY. 
[15] Chris Tisdell, What is a Lipschitz condition?, Dr Chris Tisdell's YouTube Channel, 14/01/2012, accessed 23/01/2015, www . youtube. com/watch?v=Cnc83B3C2pY. 